\title{
Metadislocations in Complex Metallic Alloys
}

\author{
Marc Heggen, Markus Heidelmann, Michael Feuerbacher \\ Ernst Ruska-Centrum \& Peter Grünberg Institut, Forschungszentrum Jülich GmbH, 52425 Jülich, \\ Germany.
}

Complex Metallic Alloys (CMAs) represent a large group of crystalline intermetallics comprising some hundreds of known phases in various alloy systems. They are characterized by the presence of icosahedral atom coordination, large lattice constants, and a correspondingly large number of atoms per unit cell, typically ranging between a few tens and some thousand [1]. In recent years, CMAs have attracted increasing research interest and exceptional properties have been found e.g. [2]. Several CMAs also show unusual plastic properties, the occurrence of new plastic-deformation mechanisms and novel defects [3]. Most prominent among those novel defects are metadislocations [3,4], which are considered the most complex defects in materials science as they involve several hundreds of atoms in their core. They are partial dislocations which are not associated to conventional planar defects but to slabs of a structurally transformed complex phase or a certain number of so-called phason planes. Metadislocations were first observed in the CMAs $\varepsilon_{6}$ - and $\varepsilon_{28}$-Al-Pd-Mn $[3,4]$, later in $\varepsilon$-type Al-Pd-Fe [5], $\mathrm{Al}_{13} \mathrm{Co}_{4}[6]$, and in T-Al-Mn-Pd [7,8].

In this contribution, the properties of metadislocations in different phases are briefly reviewed and recent microstructural investigations on their core structure using aberration-corrected high-angle annular dark field scanning transmission electron microscopy (HAADF-STEM) are presented. In the phase T-Al-Mn-Pd (Figure $1 \mathrm{a}$ ), a novel and highly complex deformation mechanism is found, which is based on the movement of a metadislocation core mediating strain and separate escort defects [7]. Upon deformation, the escort defects move ahead and locally transform the T-phase structure for accommodation of the dislocation core (Figure $1 \mathrm{~b}$ ). Dislocation core and escort defects leave a slab of a modified structure, which can be interpreted as a stack of stacking faults. Different types of metadislocation with Burgers vectors $\vec{b}=-(-\tau)^{-n} c(0,0,1)$, with $\mathrm{n}=2,3,4$ are found [8]. They are associated to two, four and six planar faults, respectively. This mechanism implies the coordinated movement of hundreds of atoms per elementary glide step. Although the mechanism is very complex, it can be described by a simple jigsaw-puzzle-like rearrangement of basic structural subunits. A very similar mechanism involving metadislocation and escort defect motion was recently found in the complex phase o- $\mathrm{Al}_{13} \mathrm{Co}_{4}$ [6]. Here, two types of dislocations with six and four stacking faults are found, respectively. In addition, a first partial atomic model for metadislocation glide motion was developed.

The largest and by far most complex types of metadislocations are observed in the phase $\varepsilon_{6}-\mathrm{Al}-\mathrm{Pd}-\mathrm{Mn}$. Due to the large number of atoms involved - a single step of motion involves structural rearrangements of about 2000 atoms per unit-cell thickness - the mechanism of motion is largely unknown. Recent analysis of the atomic structure of a metadislocation core using aberration-corrected high-resolution electron microscopy showed that the core is highly ordered and based on atomic clusters [9]. It possesses a multiscale structure with an inner core comprising the full strain of the metadislocation, and an outer core, which is not strained but has a cluster structure modified with respect to the bulk. A first partial atomic model for metadislocation motion based on repetitive jump patterns along various crystallographic directions is developed, which involves the movement of about 100 atomic positions per step of motion. Characteristic parallel 'paired jumps' of neighbouring atomic columns lead to a net 
flux of atoms towards the metadislocation core which is consistent with the negative climb mode of metadislocation motion.

[1] K Urban, M Feuerbacher, J. Non Cryst. Sol. 334 (2004), p. 143.

[2] M Armbrüster et al., Nature Materials 11 (2012), p. 690.

[3] M Feuerbacher, M Heggen, „Dislocations in Solids: Metadislocations“ Vol 16. ed. J.P. Hirth and L.

Kubin, (Elsevier, Amsterdam) p. 110.

[4] H Klein, M Feuerbacher, P Schall, K Urban, Phys. Rev. Lett. 82 (1999), p. 3468.

[5] M Feuerbacher, S Balanetskyy, M Heggen, Acta Mater 56 (2008), p. 1849.

[6] M Heggen, M Feuerbacher, Mat. Res. Lett. (2014) DOI: 10.1080/21663831.2014.882869.

[7] M Heggen, L Houben, M Feuerbacher, Nature Materials 9 (2010), p. 332.

[8] M Heggen, L Houben, M Feuerbacher, Acta Mater. 59 (2011), p. 4458.

[9] M Heggen, M Feuerbacher, Acta Mater. 61 (2013), p. 3851.
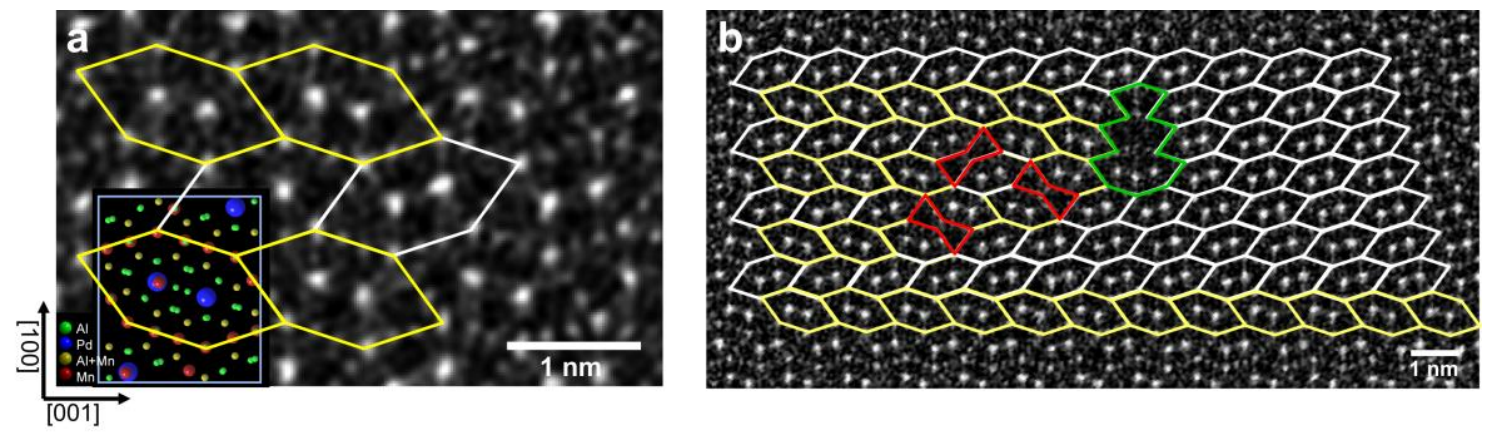

Figure 1. a) HAADF-STEM micrograph and model of T-Al-Mn-Pd along ( $\left.\begin{array}{lll}0 & 1 & 0\end{array}\right)$. The orthorhombic structure can be divided into structural subunits (white and yellow rhombs). b) Metadislocation in the T-phase. The dislocation core (green polygon) is escorted by three additional defects (red polygons). Upon movement, they leave a slab of a modified structure (parallelly oriented white rhombs) [7].
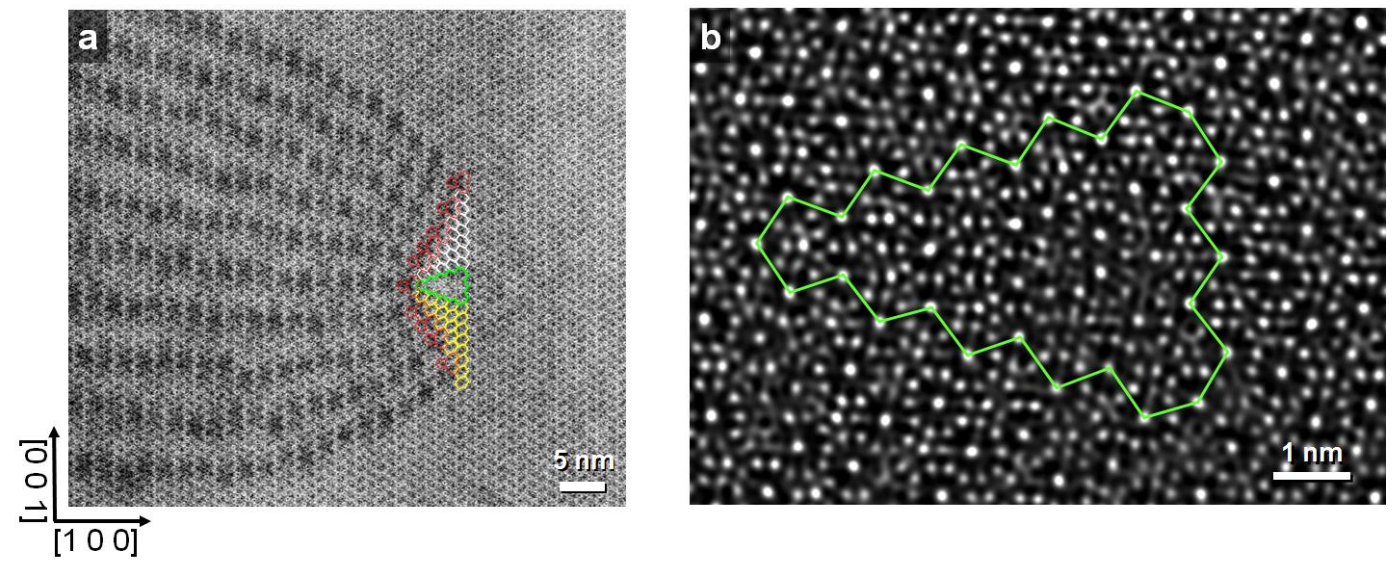

Figure 2. a) Micrograph of a metadislocation in $\varepsilon_{6}$-Al-Pd-Mn. Dark features on the left are phason planes. The core of the metadislocation is represented by a green polygon. b) HAADF-STEM micrograph of a metadislocation core (green polygon). Atomic columns occupied by $\mathrm{Pd}$ are visible as bright dots [9]. 